\title{
3-D Simulations of Plasma Transport in the Ring Limiter Scrape-Off Layer of Tokamak Aditya
}

\author{
Devendra Sharma, ${ }^{1}$ Ratneshwar Jha, ${ }^{1}$ Yühe Feng, ${ }^{2}$ and Francesco Sardei ${ }^{2}$ \\ ${ }^{1}$ Institute for Plasma Research, Bhat, Gandhinagar, India, 382428 \\ ${ }^{2}$ Max-Planck-Institut für Plasmaphysik, \\ Wendelsteinstr. 1, Greifswald, D-17491, Germany
}

(Dated: November 16, 2012)

\begin{abstract}
Aditya tokamak has a circular poloidal ring limiter at one toroidal location and therefore its contact with plasma produces a complex 3D flow pattern in the SOL plasma region [1]. Presently plasma flow in the SOL region is being measured using Mach probes at a few discrete locations and can be properly understood only with the help of a full 3D simulation taking the toroidal asymmetry introduced by the ring limiter into account. We have therefore carried out plasma transport studies for the Aditya configuration using the 3D Monte-Carlo plasma-neutral code EMC3-Eirene $[1,2]$. The toroidal asymmetry of the plasma parameters and flows indicate the importance of limiter as a localized sink of plasma. The plasma and neutral particle density show a corresponding variation in the toroidal direction as well as in its radial penetration depth. The plasma configurations are characterized by sharp gradients in the edge and show the presence of extended zones of large parallel flow shear in the SOL and edge regions. The plasma flow patterns show poloidal modulations which correspond to effects of a rational surface interacting with the limiter.
\end{abstract}




\section{INTRODUCTION}

The tokamak Aditya is a device with a major radius of $75 \mathrm{~cm}$, a minor radius of $25 \mathrm{~cm}$ and a toroidal magnetic field of $\sim 0.72 \mathrm{~T}$ on the axis. The toroidal plasma with a circular crossection has typical discharge parameters, plasma current $I_{p} \sim 75 \mathrm{kA}$, a chord-averaged central density $n_{e} \sim 1-2 \times 10^{19} \mathrm{~m}^{3}$ and the central electron temperature $T_{e 0} \sim 300 \mathrm{eV}$. The general interest of the experimental activities on Aditya tokamak has been the study of turbulent transport in the edge, including the pioneering observations on the intermittency in the edge plasma turbulence [3]. A variety of experiments on the device involve analysis and control of phenomena related to multi-scaled turbulent fluctuations of the plasma $[4,5]$. The origin of these turbulent plasma fluctuation predominantly lies in the region of the transition from a confined plasma, by well defined nested magnetic surfaces, to a rather narrow boundary called the scrape-off layer where the field lines are open and have a unique distribution of connections lengths to the plasma limiting structures.

The plasma in Aditya is limited by a limiter which has a circular shape and is covering the full poloidal circumference at a fixed toroidal location. A schematic of this set up is shown in Fig. 1. Several major factors responsible for generating the plasma (floating potential) fluctuations have been identified in various experiments on the device over last two decades, for example, the circular plasma shape resulting from the above set up is expected to have sharp gradients of $E \times B$ poloidal flow giving rise to a velocity shear layer [6]. Apart from this the fluctuations are observed to propagate in the electron diamagnetic direction inside the region of closed flux surfaces and in the ion diamagnetic direction in the SOL, indicating a distinct boundary created by the Last Closed Flux Surface (LCFS) between these two regions. The 3D topology of the plasma transport, in the region just inside the LCFS where parallel flows are weak and in the adjacent SOL where these flows are stronger and directed to the limiter along the open field lines, is therefore expected to have finite correlations with the scale length distribution of the observed fluctuations. Observation of a corresponding shear layer were recently made in the Mach probe measurements on the device [6]. In the present 3D simulations we perform analysis of such a plasma equilibrium mainly in the open field line SOL region of Aditya where the plasma flows develop from their stagnation values to reach Mach number $\sim \pm 1$. The resulting gradients of plasma parameters may affect the stationary profiles of the plasma in the closed field line edge region of the core 

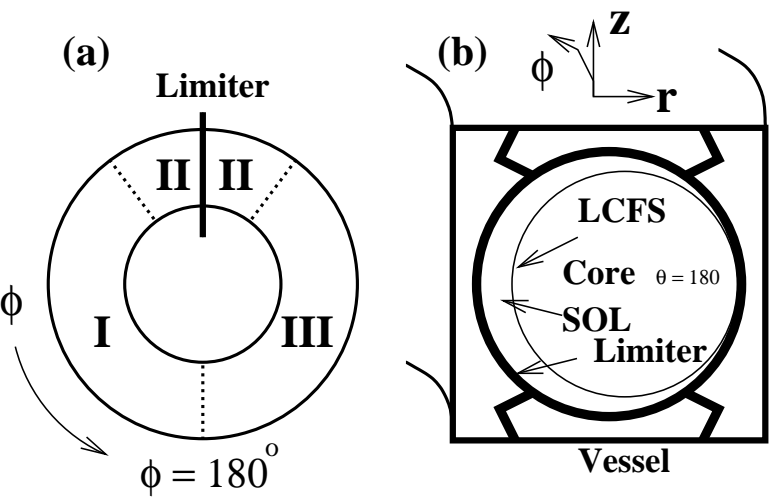

FIG. 1: Schematics of the Aditya limiter configuration, (a) top view, the zones I-III are defined for optimized 3D simulations (b) the toroidal crossection at the limiter location, $\phi=0^{\circ}$.

plasma. In order to capture these purely 3-dimensional features the present simulations are performed using the 3-dimensional SOL plasma neutral simulation code combination EMC3-Eirene [1, 2]. The EMC3 code implements a stochastic Monte-Carlo procedure to solve the collisional Braginskii fluid equations and compute transport of the plasma species. The neutral transport is simulated by the Eirene code which implements a complete kinetic model for the neutral particles.

\section{RING LIMITER SOL IN ADITYA}

In absence of an active feedback, the plasma MHD equilibrium in Aditya is, in general, diagnosed to have a Shefranov shift and has a center which is effectively shifted by a few $\mathrm{cm}$ to the outboard direction with respect to the center of the limiter, or that of the rectangular vessel, as shown in Fig. 1(b), and though this shift tends to reduce with the plasma radius, the LCFS of the configuration has a net shift in the outboard direction. In the above setup interaction of the plasma with the ring limiter results in a poloidally narrow strike region, localized on the outboard side of the circular limiter. The typical measurements of plasma position using magnetic probes indicate the position of strike region on the limiter perimeter at the outboard mid-plane with a slight up or down shift, depending on the vertical plasma stability during the discharge [6]. It is interesting to note that the 3-dimensional SOL forming in this setup has two distinct regions or volumes, first with very short connection length, not exceeding $2 \pi\left(R+r_{0}\right)$, where $R$ is the major radius and $r_{0}$ is the inner radius of 
the ring limiter, and second with considerably longer connection lengths. In particular, they correspond respectively to a volume of the SOL beyond the limiter radius $r_{0}$ and a volume of SOL which is bounded by the LCFS and the limiter radius. The latter can be identified as the volume of rotation of the extension of the crescent shaped region between the LCFS and the limiter ring about the major axis, in which the rational $n>m$ surfaces are still likely to have reasonably longer field lines which intersect the limiter only in the outboard region after making $\leq n / m$ toroidal turns.

In the present simulations using EMC3-Eirene we find the evidence of stationary flow velocity structures extended along the open field lines intersecting the limiter, that result in finite modulation of flow velocity along the poloidal direction $\theta$ owing to $q>1$. The interaction of these stationary structures with stronger drift and current generated flows, e.g. the diamagnetic and Pfirsch-Schlueter flows routinely observed in the device [6], needs to be investigated further to explore its possible correlations with the observed fluctuations. The flow patterns in the present simulations however emerge from the residual boundary flows to the limiter but are expected to be considerably magnified in magnitude as they have origin in the $q$ value profiles that are essentially unchanged in the realistic drift dominated discharges. Since the toroidally localized ring limiter breaks the toroidal symmetry of the plasma, in presence of a finite loop voltage preferential flows of the plasma species develop along the field lines incident on two different toroidal sides of the limiter, identical to that on the two faces of a Mach probe receiving differential currents in a toroidally drifting or a current carrying plasmas. The toroidal variations in the vicinity of the limiter are thus as equally dominant as the radial and poloidal gradients present at any other toroidal location in the region of LCFS.

\section{THE SIMULATION SET-UP}

In the present implementation of the code EMC3-Eirene, a 3-dimensional mesh is used covering the complete toroidal poloidal and radial extension of the device SOL. The toroidal extension of the mesh $0<\phi<360$ is further subdivided in three simulation zones as described schematically in the Fig. 1(a). This subdivision is required for two reasons, first, the mapping technique adopted in EMC3 requires convex helical cells [7] which is possible only for limited field line length, second, the recycling region around the limiter needs finer 

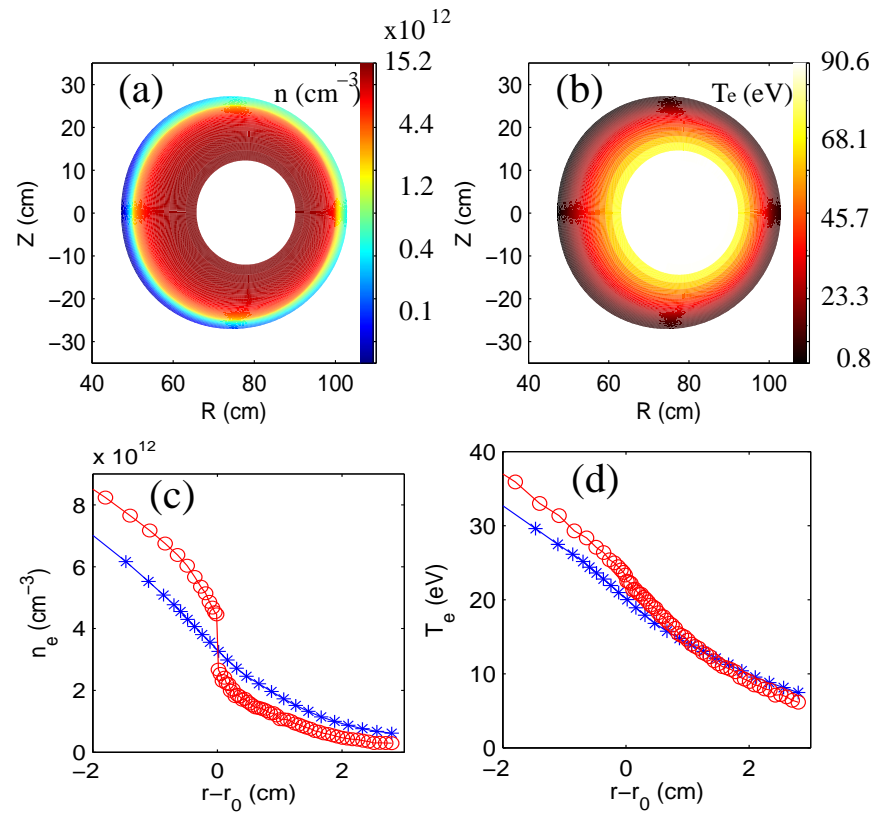

FIG. 2: Plasma density (a) and electron temperature $T_{e}(\mathrm{~b})$ distribution on radial-poloidal cell plane at the toroidal location $\phi=0^{\circ}$ close to the limiter. Radial distribution of the plasma density (c) and the electron temperature (d) on the outboard mid-plane at toroidal locations $\phi=180^{\circ}(*)$ and $\phi=0^{\circ}(\mathbf{o})$.

resolution. The ion collecting face of the limiter is located at the center of zone-II, i.e., at toroidal location $\phi=0^{\circ}$. The 3-D mesh consists of 2-D arrays of nodes, extending along the toroidal and poloidal dimensions of each of the radially separated nested magnetic flux surfaces that are derived from a magneto-hydrodynamic (MHD) equilibrium computed for the device using the equilibrium code EFIT.

The SOL plasma transport simulations in EMC3-Eirene uses boundary conditions in the form of density value at the core-SOL interface and the plasma flow velocity at the target boundaries where the field lines are incident on the target. The parallel flow velocities of the species are accordingly set to be the ion acoustic speed of the species $\mathrm{H}^{+}$for the present simulations. The parallel transport coefficients have their classical values in the underlying collisional fluid model of the plasma, the particle and heat diffusion across the magnetic field are anomalous and are empirically set to be $D=1 \mathrm{~m}^{2} / \mathrm{s}$ and $\chi=3 D$ for the present computations. An effective radial decay length is used on the radial boundary of the plasma other than targets. The net power across the core-SOL interface is a user input too, and is set in a range of $60-600 \mathrm{~kW}$ in various cases simulated. This net power input determines 
the scale of the overall 3-dimensional electron and ion temperature profiles and controls the ionization profiles via various atomic and molecular processes modeled in the Monte-Carlo neutral code Eirene which is coupled with the plasma computations done by the code EMC3. In the vacuum region beyond the SOL boundary a coarser mesh is used to sample the neutral particle distributions determined by Eirene. Various sets of computations are performed to analyze the tentative equilibrium profiles of plasma parameters, namely, plasma density, electron and ion temperatures and plasma flow velocities with a range of input parameters, largely the density at the core-SOL interface and the input power. In the present paper we however limit the analysis to that of the simulations results obtained with a fixed moderate value of the total input power, $60 \mathrm{~kW}$, from the core, and the density at the LCFS $\sim 2 \times 10^{12}$ $\mathrm{cm}^{-3}$.

\section{PLASMA DISTRIBUTIONS AND 3D FLOW STRUCTURES}

A simplistic scenario is analyzed in the present simulations by implementing an idealized, completely recycled plasma without external particle sources. The plasma density and temperature profiles in the edge and SOL regions are plotted in Fig. 2(a) and (b), respectively, at toroidal location $\phi=0^{\circ}$ exhibiting sharper gradients in the transition region localized at the LCFS as compared to the radial locations away from the LCFS. A region of large radial density gradient is seen to be present in the outboard midplane radial density profiles extracted from the radial-poloidal plane located before $\phi=0^{\circ}$ which is the toroidal location of the limiter surface. This is in contrast to the density profile extracted from the stagnation location $\phi=180^{\circ}$ where the density drop is seen to occur at a much larger radial scale length. The effect is stronger in the radial profile at the outboard midplane which corresponds to the poloidal location of the limiter and is presented in Fig. 2(c). This indicates that both poloidal and toroidal variations exist in the density distribution in the SOL region. The radial electron temperature profiles at the outboard midplanes for $\phi=0^{\circ}$ and $180^{\circ}$ however show similarity due to the dominance of parallel energy transport as presented in Fig. 2(d).

The Mach number profiles presented in Fig. 3 exhibit interesting trends where the profile close the ion collecting face of the limiter in Fig. 3(b) shows a clear effect of the vicinity of the limiter as plasma flow velocity along the field lines incident on the limiter develop 

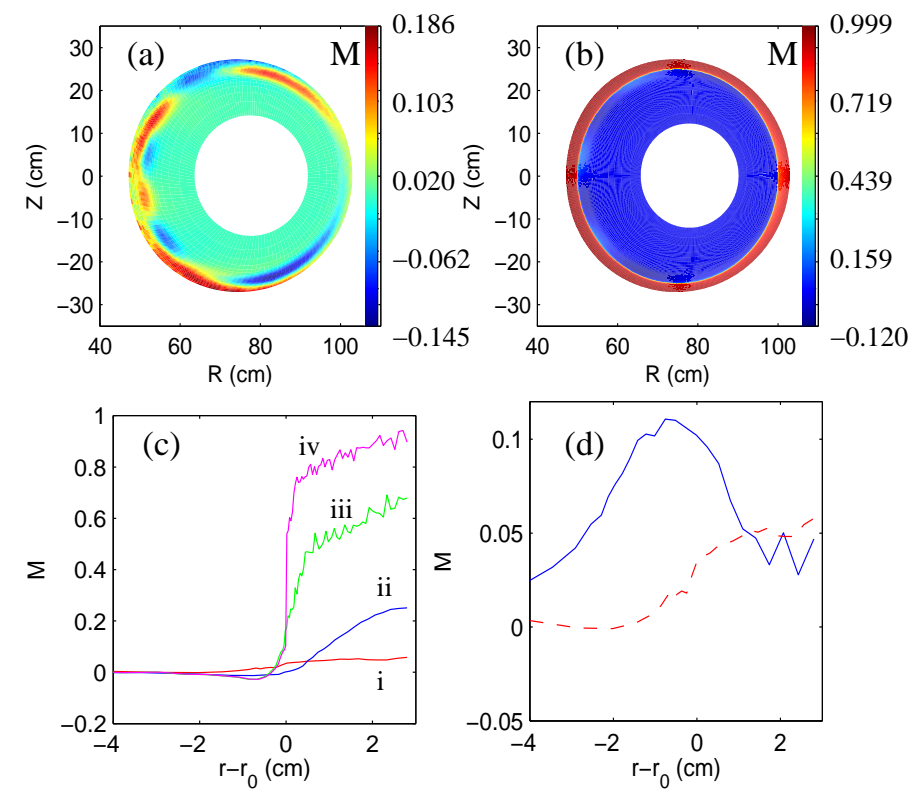

FIG. 3: The radial poloidal distribution of the plasma Mach number at toroidal cross-sections located at (a) $\phi=180^{\circ}$ and (b) $\phi=0^{\circ}$, i.e., crossection approaching the ion collecting face of the limiter. (c) The radial variations of the plasma Mach number, i, ii, iii and iv, along the outboard mid-plane at toroidal locations $\phi=180^{\circ}, 90^{\circ}, 12^{\circ}$ and $0^{\circ}$, respectively. (d) Radial profiles of Mach number along the inboard (solid line) and outboard midplanes (broken line) at toroidal location $\phi=180^{\circ}$.

parallel gradients to nearly approach its boundary value $M=1$. Fig. 3(b) shows the Mach number distribution on the toroidal face of the limiter where values at the cell centers before the limiter surface are plotted. The limiter form can be identified by the sharp increase in $M$. The slight deviation in $M$ from unity is the finite cell size effect. This deviation is little larger on the outboard side because the most of the recycling takes place there. On the other face of the limiter $M$ exhibits a similar distribution pattern, though with a reversed flow direction. One would expect that the flow should vanish at $\phi=180^{\circ}$ plane opposite to the limiter location. In contrast to this, the 3D simulations show that there do exist finite flow with complex structures (see Fig. 3(a)). In fact, the existence of such flows is already evident in the core region near the LCFS in Fig. 3(b) where the core and SOL flows have opposite directions. The ionization takes place within a narrow zone strongly localized in both poloidal and toroidal directions. The resulting $\mathrm{H}$ ions escape from the recycling region and disperse on the flux surfaces mainly through convection along the field lines before 


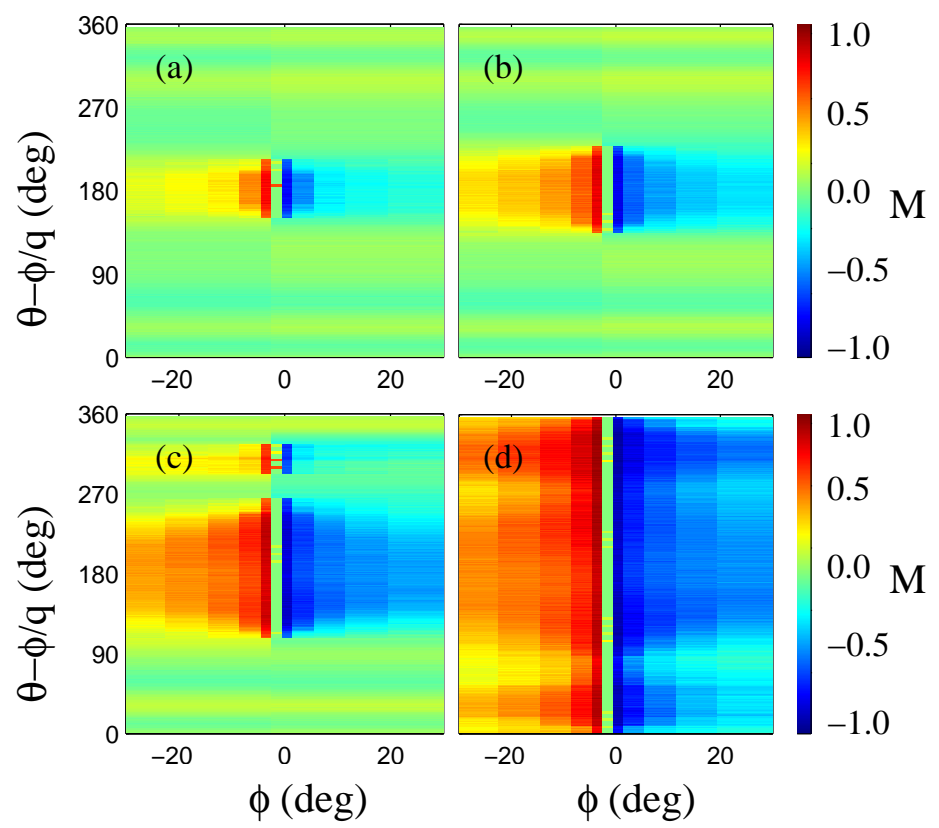

FIG. 4: The distribution of plasma Mach number on toroidal-poloidal magnetic surfaces with increasing radius in zone-I. Plots (a) - (d) correspond to radial index, $n_{r}=28,35,45$ and 52 respectively, the circular outer radial boundary of the simulation zone corresponds to $n_{r}=72$ for zone-II. The lighter patch at $\phi=0$ represents the limiter ring.

diffusing into SOL and finally flowing back to the limiter. In the case of a rational surface interacting with the limiter, the convection on the surface is stronger along the field lines that lead to the narrow strike zone at the limiter resulting in the structures and poloidal modulation seen in the flow profiles. The rest of the field lines on the surface are close and form zones of weaker parallel flow.

Influence of this complex SOL topology is much more elaborate when the flow profiles along individual magnetic flux surfaces are examined. These surfaces are represented as a set of nested toroidal-poloidal surfaces, separated radially with each other. The surfaces radially beyond the LCFS have a limited poloidal intersection with the ring limiter which increases with the radius of each toroidal surface. Beyond a certain value of the radius the surfaces begin to intersect the limiter along their entire poloidal extension. While the first kind of surfaces with partial poloidal limiter intersection form the volume with longer connection length, the later kind of surfaces constitute the volume where the connection lengths are at most $2 \pi\left(R+r_{0}\right)$ for $q \geq 1$. We examine the parallel Mach number profiles in the simulations along these surfaces in Fig. 4 where the toroidal-poloidal profile of the Mach number is 
presented with increasing radial index of the surfaces. The surfaces show toroidal plasma flows developing that satisfy the boundary values at the intersection of these surfaces with the ring limiter at the strike point located at the outboard side at $\theta \sim 180^{\circ}$. An additional strike point at the limiter is consequence of change in grid resolution where a larger cell has been identified as a limiter cell since its center lies behind the limiter surface. The radial variation in the toroidal Mach number for the plasma is presented at various values of $\phi$ in Fig 3(c) which show development of a strong toroidal shear layer at the LCFS location for $\phi$ approaching the limiter surface. The radial modulations can be seen best in the profiles at the toroidal locations away from the limiter where the flows become weak as seen in Fig 3(d). A close correspondence between the profiles in Fig 3 and the radial variation of the Mach number measured in the device exists as sharp radial gradient in Mach number is also measured in the region of LCFS in Aditya [6].

Note that in the simulations the stronger parallel flows, directed to the limiter, are driven predominantly by the $\nabla p$. Since the effects of plasma drifts are excluded form the present computations the contributions from radial or poloidal drift generated flows to these parallel flows are absent. Such contributions are expected to be significant and could dominate the parallel flows, which in present simulations are driven purely by diffusive particle source from the core, particularly at large $|\phi|$ (i.e., close to the stagnation point) where the plasma flow velocity vanish owing to the symmetry considerations. Since the flow structures observed have their origin in the background plasma equilibrium their topology must be largely unaffected by values of the background density and the additional flows which, though excluded from the present simulations, are usually significant in the experiments [6]. The present simulations thus highlight impact of the setup where despite the poloidal continuity of the limiter the magnetic surfaces located beyond the LCFS are not completely open and only a small poloidal extension of them intersect the limiter. One radially visible consequence of this is that because of the filamentation of the parallel flows directed to the limiter in the SOL the flow profiles and the shear in the edge region are no longer poloidally continuous (in $\theta$ ). The poloidal drifts and flows in the device can therefore be expected not to be divergence free which must affect their ability to produce an effective transport barrier [8] for the plasma. However, the exact mechanism how the observed structures interact with the drift and flows, that are excluded from the present simulations, and the issue that whether an interaction of flow structures with finite poloidal currents can cause additional radial 
transport in the SOL via larger spatial scale structures like plasma blobs [9], need to be investigated in further detail.

\section{SUMMARY AND CONCLUSIONS}

The first 3D simulations of the plasma transport in the SOL of tokamak Aditya, generated by a toroidally localized poloidal ring limiter, was performed using plasma neutral transport code EMC3-Eirene. The simulations show 3-dimensional structure of the flow patterns in the SOL that has a distinct boundary between the regions of shorter and comparatively longer connection lengths on a partly open magnetic surface. Rational magnetic flux surfaces interacting with the limiter, in combination with diffusive cross-field plasma transport in the region of long connection length, produces zones with poloidal modulations of the flow velocity. The simulations results show correspondence with the observation in the device of a parallel flow shear across the LCFS [6] whose impact on the confinement properties of plasma needs to be investigated further in view of its 3D topology as seen in the present simulations.

[1] Y. Feng, F. Sardei, and J. Kisslinger, J. Nucl. Mater 266-269, 812 (1999).

[2] D. Reiter, Tech. Rep. Technical Report Juelich-1947, KFA, Juelich, Germany (1984).

[3] R. Jha, P. K. Kaw, S. K. Mattoo, C. V. S. Rao, Y. C. Saxena, and A. Team, Phys. Rev. Lett. 69, 1375 (1992).

[4] R. Jha, A. Sen, P. K. Kaw, P. K. Atrey, S. B. Bhatt, K. Tahiliani, R. L. Tanna, and A. Team, Plasma Phys. Control. Fusion 51, 095010 (2009).

[5] K. Tahiliani, R. Jha, M. V. Gopalakrishna, K. Doshi, V. Rathod, C. Hansalia, and A. Team, Plasma Phys. Control. Fusion 51, 085004 (2009).

[6] D. Sangwan, R. Jha, J. Brotankova, and M. V. Gopalkrishna, Phys. Plasmas 19, 092507 (2012).

[7] Y. Feng, F. Sardei, and J. Kisslinger, Phys. Plasmas 12, 052505 (2005).

[8] F. Wagner et al., Phys. Rev. Lett. 53, 1453 (1984).

[9] N. Bisai, A. Das, S. Deshpande, R. Jha, P. K. Kaw, A. Sen, and R. Singh, Phys. Plasmas 11, 4018 (2004). 